\title{
ASTEROID MASS DETERMINATION: (1) CERES
}

\author{
M. CARPINO \\ Osservatorio astronomico di Brera, Milano
}

AND

Z. KNEŽEVIĆ

Astronomska opservatorija, Beograd

Recent improvements of observational accuracy, stellar catalogues and efficiency of the procedures for searching for asteroid close encounters (Kuzmanoski and Knežević, 1993) gave rise to an increased interest in the problem of determination of asteroid masses (Bowell et al., 1995).

We have made an attempt to determine the mass of the largest asteroid (1) Ceres by analysing variations due to close approaches to Ceres in the orbits of a total of 9 minor planets. The list of our objects includes all the asteroids used in previous attempts, as well as some additional ones for which potentially favourable close encounters were found by Kuzmanoski and Knežević (1994). The method we used consists in the simultaneous determination of the corrections to the orbital elements of the perturbed asteroid and of the mass of Ceres by employing a standard least square fit. The dynamical model included major planets from Mercury to Neptune, (2) Pallas and (4) Vesta. In particular the inclusion of Vesta proved to be important in some cases. For the planets we used initial conditions and masses from JPL DE200 ephemeris, while for Pallas and Vesta we used masses of $1.14 \times 10^{-10}$ and $1.33 \times 10^{-10} M_{\odot}$ (Goffin, 1991). Observations were obtained from the Computer Service of the Minor Planet Center.

The results are summarized in Table 1, which is sorted in order of increasing formal RMS error of the derived mass. The RMS errors of the observations with respect to the fit (square root of the average value of $\left.\Delta \alpha^{2} \cos ^{2} \delta+\Delta \delta^{2}\right)$ range from 1.0 to 1.8 arcsec, and depend strongly upon the criterion chosen for outlier rejection. Masses found in the first five cases show a satisfactory mutual agreement: their weighted average amounts to $(4.67 \pm 0.09) \times 10^{-10} M_{\odot}$, which is quite close to the values found in the most recent determinations based on close-approach analysis (Goffin, 1991; 
TABLE 1. Summary of the results. The columns contain: asteroid number, date of the close approach with Ceres, product of the close encouter distance $\rho$ and velocity $\Delta v$ (proportional to the reciprocal velocity change produced by the close approach), number and timespan of observations before $\left(N_{-}, \Delta T_{-}\right)$and after $\left(N_{+}, \Delta T_{+}\right)$the close approach, inferred mass $(M)$ and its RMS error $(\delta M)$

\begin{tabular}{|c|c|c|c|c|c|c|c|c|}
\hline Ast. & $\begin{array}{l}\text { Cl. App. } \\
\text { MJD }\end{array}$ & $\begin{array}{c}\rho \Delta v \\
10^{-5} \mathrm{AU}^{2} / \mathrm{d}\end{array}$ & $N_{-}$ & $\begin{array}{c}\Delta T_{-} \\
\mathbf{y}\end{array}$ & $N_{+}$ & $\begin{array}{c}\Delta T_{+} \\
\mathbf{y}\end{array}$ & $\begin{array}{l}M \\
10^{-10}\end{array}$ & $\begin{array}{r}\delta M \\
{ }^{\delta} M_{\odot}\end{array}$ \\
\hline (203) & 32785.26 & 3.9 & 26 & 68.9 & 70 & 45.4 & 4.77 & 0.07 \\
\hline (348) & 45944.76 & 2.0 & 47 & 91.8 & 60 & 7.9 & 4.77 & 0.14 \\
\hline (324) & 31180.67 & 10.9 & 68 & 52.0 & 520 & 49.0 & 4.54 & 0.16 \\
\hline$(91)$ & 41937.58 & 6.3 & 140 & 80.5 & 58 & 19.5 & 4.25 & 0.17 \\
\hline$(534)$ & 42769.86 & 3.6 & 46 & 71.7 & 18 & 16.3 & 4.19 & 0.36 \\
\hline$(2572)$ & 41036.47 & 3.3 & 2 & 21.1 & 31 & 21.5 & 3.38 & 0.43 \\
\hline (32) & 42741.21 & 6.8 & 144 & 105.2 & 62 & 17.2 & 5.69 & 0.50 \\
\hline$(3643)$ & 41571.14 & 1.3 & 6 & 34.8 & 14 & 17.1 & 6.50 & 0.90 \\
\hline$(2660)$ & 44343.28 & 5.7 & 4 & 55.5 & 30 & 13.9 & 18.17 & 2.14 \\
\hline
\end{tabular}

Williams, 1992; Bowell et al., 1995) as well as to the value of $4.64 \times 10^{-10} M_{\odot}$ adopted in the latest JPL DE403 Ephemeris (Standish et al., 1995). The other four cases deviate significantly from this value, exhibiting in the same time larger formal errors, due to different reasons:

(2572) Annschnell: the five critical pre-encounter observations collected in 1950 are of poor quality, and at least three of them have certainly to be discarded. A fit employing the remaining two observations produces the very low mass value given in the table; by discarding another observation we obtain $4.15 \times 10^{-10} M_{\odot}$, in better agreement with other more favourable cases. However, fit residuals alone do not give sufficient information to decide which observation should be discarded.

(32) Pomona: available data are not very sensitive to Ceres' mass, since the encounter was not very close and occured comparatively recently.

(3643) 1978 UN2: although the close encounter appeared very promising, there is only one available pre-encounter observation (made in 1937) which is far enough from the close approach date. Thus, the value of the mass depends critically on this observation, the accuracy of which cannot be assessed by statistical methods.

(2660) Wasserman: the orbit is poorly determined due to unfavourable distribution of observations, especially after the close encounter. This is revealed by high correlation coefficients between the orbital elements and, consequently, between the orbital elements and the inferred mass.

In order to infer which are the observations having larger impact on the 


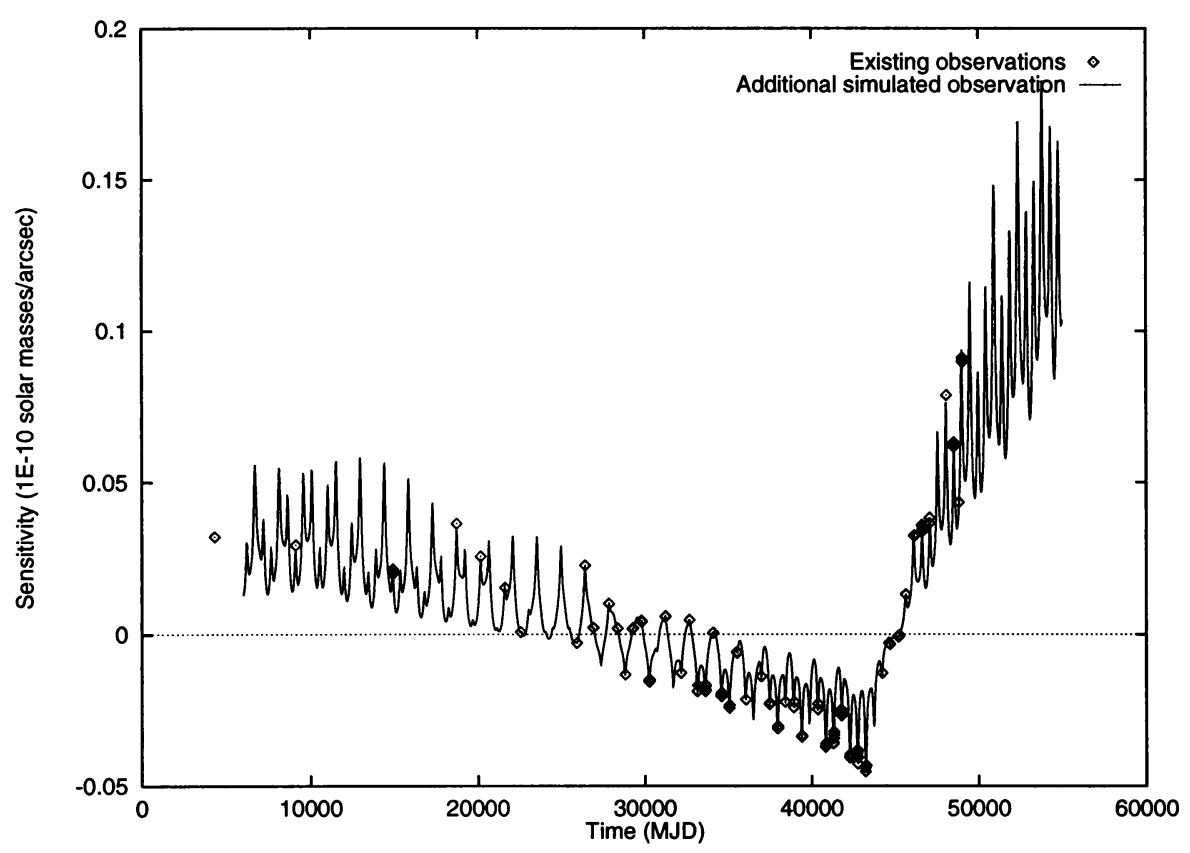

Figure 1. Sensitivity of the solution for the mass of Ceres with respect to the right ascensions of actual observations of (32) Pomona used in the fit (rhombs), and with respect to a fictitious additional observation (solid line)

mass determination, we computed the sensitivity of the fitted mass with respect to each observation, as well as to a possible additional measurement; in the latter case we added a fictitious observation and computed the least square fit using this enlarged data set, repeating the computation for a number of arbitrary instants of time within the time span covered by actual observations. The sensitivity is obtained as the partial derivative of the least square solution for the mass with respect to right ascension and declination. An example of this computation is given in Figure 1 for asteroid (32) Pomona.

The plot shows that post-encounter observations have larger influence on the mass, and hence suggests that additional future observations can significantly improve the result. Since this situation is not uncommon among the cases we have analyzed, we performed a series of simulations in order to establish whether future astrometric measurements (obtained for instance during a dedicated observational campaign) could improve the precision of the mass determination. To this purpose, we repeated the fits by adding fictitious observations distributed around oppositions in the next 10 years (1995-2005). The total number of new observations varied from 104 to 117 (depending on the asteroid). Their assumed accuracy was 0.5 arcsec in 
right ascension and declination, namely 0.71 arcsec in spherical distance; however, since we assigned equal weights to all the observations, the improvement in the error bar for Ceres mass results from the number and distribution of new data points rather than from their precision. In the case of (32) Pomona, the RMS error for Ceres mass obtained from the simulation is $0.21 \times 10^{-10} M_{\odot}$ (2.4 times smaller than the one obtained from the analysis of existing observations): this result would place Pomona among the cases which can give some information on the mass of Ceres, though not among the most precise ones. A substantial improvement of the result can be obtained also for (203) Pompeja (the error in the mass decreases by a factor 1.7), (348) May (factor 2.7), (91) Aegina (factor 2.2) and (534) Nassovia (factor 3.9 ). New observations contribute very little to the mass determination in the case of (324) Bamberga (RMS reduced by a factor 1.1): this is not surprising, since for this object astrometric observations after the date of the close approach with Ceres are already quite numerous. Although a significant increase in the formal precision can be obtained also for (3643) 1978 UN2 (by a factor 3.2) and (2660) Wasserman (factor 6.0), the resulting error bar remains anyway too large for the mass to be usable. As already noticed, a sufficient accuracy in the cases of (2660) Wasserman and (2572) Annschnell cannot be reached only by adding future astrometric data, but requires also good additional pre-encounter observations which could be possibly discovered on archive plates.

The results of the previous simulations can be summarized as follows: if we take into account the increased precision in the determination of the orbits of (203) Pompeja, (348) May, (91) Aegina, (534) Nassovia and (32) Pomona which can be achieved by a dedicated observing campaign lasting 10 years (as assumed above), we estimate an improvement in the formal error for the mass of Ceres from the present value of $9.1 \times 10^{-12}$ to $4.6 \times 10^{-12} M_{\odot}$.

\section{References}

Bowell, E., Muinonen, K. and Wasserman, L.H. (1995) Asteroid mass determination from multiple asteroid-asteroid encounters, Proceedings of Mariehamn, in press

Goffin, E. (1991) The orbit of 203 Pompeja and the mass of Ceres, Astron. Astrophys. 249, 563-568

Kuzmanoski, M. and Knežević, Z. (1993) Close encounters with large asteroids in the next 50 years, Icarus 103, 93-103

Kuzmanoski, M. and Knežević, Z. (1994) Asteroid close encounters and mutual perturbations, Planet. Space Sci. 42, 297-299

Standish, E. M., Newhall, X X, Williams, J. G. and Folkner, W. M. (1995) JPL Planetary and Lunar Ephemerides, DE403/LE403, JPL IOM 314.10-127

Williams, G.V. (1992) The mass of (1) Ceres from perturbations on (348) May, in Harris A.E. and Bowell E. eds., Asteroids, Comets, Meteors 1991, Lunar and Planetary Institute, Houston, Texas, 641-643 